\title{
Patterns and characterization of COPD exacerbations using real-time data collection
}

\author{
This article was published in the following Dove Press journal: \\ International Journal of COPD \\ 25 January 2017 \\ Number of times this article has been viewed
}

\author{
Stanley I Ejiofor ${ }^{1,2}$ \\ Jan Stolk ${ }^{3}$ \\ Pablo Fernandez ${ }^{4}$ \\ Robert A Stockley ${ }^{1,2}$ \\ 'Centre for Translational Inflammation \\ Research, University of Birmingham, \\ ${ }^{2}$ ADAPT Project, University Hospital \\ Birmingham, Birmingham, UK; \\ ${ }^{3}$ Leiden University Medical Centre, \\ Leiden, the Netherlands; ${ }^{4}$ Independent \\ consultant, Penn, UK
}

Introduction: Patients with chronic obstructive pulmonary disease often experience exacerbations. These events are important as they are a major cause of morbidity and mortality. Recently, it has been increasingly recognized that patients may experience symptoms suggestive of an exacerbation but do not seek treatment, which are referred to as unreported or untreated exacerbations. Symptom diaries used in clinical trials have the benefit of identifying both treated and untreated exacerbation events.

Methods: The Kamada study was a multicenter, double-blind randomized controlled trial of inhaled augmentation therapy in alpha-1 antitrypsin deficiency (AATD). A retrospective review of daily electronic symptom diary cards was undertaken from the two leading centers to identify symptomatic episodes consistent with a definition of an exacerbation. The aims were to explore the relationship between exacerbation events and classical "Anthonisen" symptoms and to characterize treated and untreated episodes.

Results: Forty-six AATD patients with airflow obstruction and history of exacerbations were included in the analysis. Two hundred thirty-three exacerbation episodes were identified: 103 untreated and 130 treated. Untreated episodes were significantly shorter (median 6 days; interquartile range [IQR] 3-10 days) than the treated episodes (median 10 days; IQR 5-18.25 days: $P<0.001)$. Using logistic regression analysis, Anthonisen type and length of dyspnea were significant predictors of the treatment of an exacerbation event.

Conclusion: Real-time electronic diary cards provide valuable information about the characterization of exacerbations. Untreated episodes are common and are significantly shorter in duration than the treated episodes. Dyspnea is the most important single Anthonisen symptom in the prediction and/or driver of treatment.

Keywords: exacerbations, alpha-1 antitrypsin deficiency, COPD

\section{Introduction}

Patients with COPD often experience exacerbations defined as an acute deterioration in respiratory symptoms beyond normal day-to-day variability that leads to a change in medical therapy. ${ }^{1}$ These events are important as they are a major cause of morbidity and mortality. ${ }^{2-6}$ The frequency of exacerbations has been associated with worse health status, ${ }^{3}$ increased mortality risk, ${ }^{7}$ and accelerated lung function decline. ${ }^{8,9}$ As a consequence, the reduction and prevention of exacerbations by therapeutic interventions are often studied with the aim to change the course of COPD.

The classical and widely used characterization of exacerbations was initially described by Anthonisen et $\mathrm{al}^{10}$ and became universally referred to as the "Anthonisen criteria" for the definition used in most scientific and clinical publications. With this overall clinical definition, subsequent studies assessed exacerbation severity by the degree of therapeutic intervention required and health care resources used. ${ }^{11-13}$
Correspondence: Robert A Stockley University Hospital Birmingham, ADAPT Project, Respiratory Department, Mindelsohn Way, Birmingham, BI5 2GW, UK Email rob.stockley@uhb.nhs.uk 
However, over recent years, a greater insight into the nature of exacerbations has emerged, largely from the use of patient recall and manual or electronic symptom diary cards. . $^{3,6,8,14}$ The ability of diary cards to identify treated (self-perceived changes in Anthonisen [AT] symptoms receiving treatment) and untreated (AT symptom changes suggesting exacerbation but not leading to a change in treatment) exacerbations has raised uncertainty about the importance of some of these symptoms if treatment was not required or taken. These untreated episodes are, however, common and likely to be detrimental to lung function and health status, ${ }^{3,6,8,14}$ raising the question of whether they should also be treated. The episodes are reported to be shorter in length and associated with an early improvement in "well-being" prior to the time when usual therapy is sought/instigated. ${ }^{6}$

While the understanding of exacerbation events has improved, the pattern of patient symptoms during exacerbations and, in particular, the relationship between classical AT symptoms and treatment have not been established. The recent study of inhaled AAT was a double-blind randomized controlled trial in COPD patients with alpha-1 antitrypsin deficiency (AATD) and a documented history of frequent exacerbations. Patients enrolled in the study completed daily electronic symptom diary cards prompted by daily electronic reminders. The diary cards were reviewed by two of the authors (PF and RAS) to identify episodes consistent with a definition of an exacerbation. The aims were to explore the relationship between exacerbation events, the pattern of AT symptoms, and the relationship to treatment.

\section{Methods}

The Kamada study (ClinicalTrials.gov; NCT01217671) was a multicenter, double-blind randomized controlled trial of inhaled augmentation therapy in AATD. Ethical approval for this present study and the Kamada study was provided by UK REC Nottingham Ethics Committee 09/H0408/103 and the Ethics Committee of Leiden University Medical Centre, with all participants providing consent for the study and for their anonymized data to be used in other studies. A retrospective review of daily electronic symptom diary cards from the two leading participating centers with most patients recruited was conducted prior to trial unblinding. All patients included were sequentially enrolled and had follow-up from the time of enrollment for at least 1 year. Any patient with $<1$-year follow-up was not included. The electronic diary contained details of all AT symptoms and change of medical therapy (additional corticosteroids, antibiotics, and bronchodilators). Scoring of AT symptoms in the electronic diary is that of the manual Bronkotest (Middlesex, UK) and described previously. ${ }^{6}$
In summary, dyspnea was scored on a 4-point scale ( $2=$ normal or usual for me, $3=$ worse than usual, $4=$ much worse than usual, and $1=$ better than usual), daily sputum volume was scored as none $=(0), 1=($ up to a teaspoonful $), 2=($ up to a tablespoonful $)$, $3=$ (up to an egg-cupful), 4= (more than an egg-cupful), and sputum color was assessed using the Bronkotest color chart (1-2 being mucoid and 3, 4, and 5 being increasing purulence).

The daily diary printout was initially reviewed independently by two physicians (RAS and PF), and where any differences were observed the episode was reviewed jointly to obtain a consensus. Exacerbations were identified when one or more major AT criteria deteriorated from a baseline period of stability for $>2$ consecutive days. ${ }^{13,15}$ The method for identifying the start and end of exacerbations was as those published previously. ${ }^{6}$ In addition, the duration of deterioration for each AT symptom from the patient's baseline or "usual" state during the exacerbation period was noted. Symptoms relapsing within 7 days of a previously identified exacerbation were judged to be part of the same exacerbation event.

Chronic bronchitis phenotype was defined as those characteristics that the patients with a persistent daily productive cough indicated in their electronic diary for $>3$ months of the observation period.

\section{Statistical analysis}

SPSS Version 22 (IBM Corp, Armonk, NY, USA) was used for statistical analysis. Nonparametric data are presented as median and interquartile range (IQR). Differences between treated and untreated exacerbations were compared with descriptive statistics using Mann-Whitney $U$ tests (single tailed) for continuous variables and $\chi^{2}$ or Fisher's exact test for categorical variables. Binary logistic regression analyses were then conducted to analyze the effect of major AT symptoms and AT type on treatment using all exacerbation episodes. In the logistic regression analyses AT type was coded as a categorical variable with AT type 3 (only one of the three major symptoms present) as the reference category.

\section{Results}

A total of 46 patients with AATD and airflow obstruction (forced expiratory volume in 1 second/forced vital capacity ratio $<70 \%$ ) from the two centers were included in the review. The demographic details are summarized in Table 1. Two hundred thirty-three exacerbation episodes were identified, of which 103 were untreated and 130 treated.

\section{AT type and symptom length of episodes}

Untreated episodes had a median duration of 6 days (IQR $3-10$ days $)$ and were significantly shorter $(P<0.001)$ than 
Table I Demographic details of trial participants

\begin{tabular}{ll}
\hline Age (years) & $53.80( \pm 9.65)$ \\
Male:female & $30: 16$ \\
FEV $_{1} \%$ & $42.33( \pm 14.5)$ \\
FEV $_{1} /$ FVC ratio & $33.04( \pm \mathrm{II} .05)$ \\
Chronic bronchitis & 32 \\
\hline
\end{tabular}

Note: Data shown as mean $( \pm \mathrm{SD})$ or number.

Abbreviations: $\mathrm{FEV}_{1}$, forced expiratory volume in I second; FVC, forced vital capacity; SD, standard deviation.

treated episodes (median 10 days; IQR 5-18.25 days) as summarized in Figure 1. Untreated episodes were characterized by both a shorter duration of dyspnea and sputum volume though not sputum purulence (Table 2). Of the total 233 exacerbation events, 66 were AT type 1, 64 AT type 2 , and 103 AT type 3 . There was a significant association between AT type and treatment $(P<0.001)$ with a higher proportion of AT type $1(83 \%)$ but lower proportion of AT type 3 events (35\%) receiving treatment (Figure $2 \mathrm{~A}$ ). AT type 3 events were further evaluated categorizing each episode by the symptom (ie, if sputum purulence was the clinical symptom that changed, it was labeled a purulent predominant type 3 exacerbation). Of the 103 AT type 3 exacerbations the proportions with symptom predominance for dyspnea, sputum volume, and sputum purulence were $72.8 \%, 11.7 \%$, and $15.5 \%$, respectively. There was a significant association between symptom predominance and treatment in the AT type 3 exacerbations ( $P=0.003$ Fisher's exact test). Within the three groups, the proportion of dyspnea-predominant type 3 episodes that were treated was significantly greater (43.6\%) than those due to changes in sputum volume $(16.6 \%)$ or sputum purulence $(6.2 \%)$ as summarized in Figure 2B $(P<0.05)$.

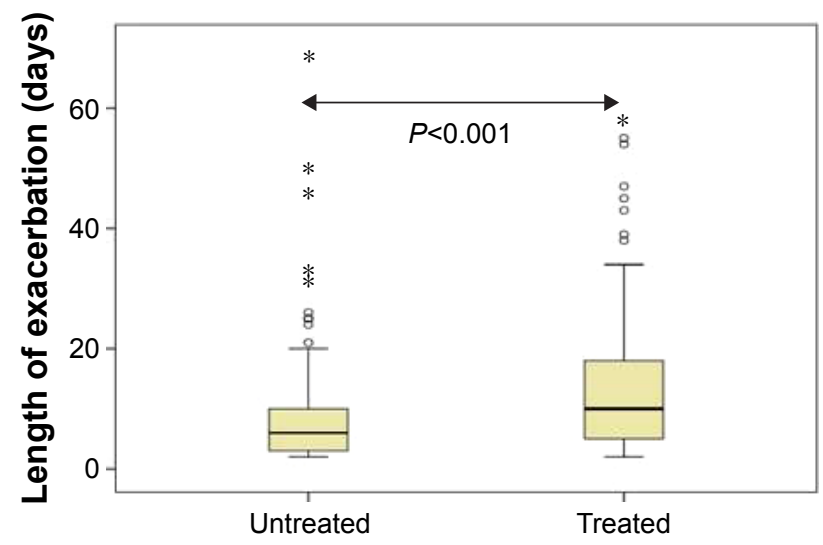

Figure I Length of untreated and treated exacerbations is shown as a box and whisker plot.

Notes: The bold horizontal line is the median value. The box is the interquartile range and the bars represent the $95 \%$ confidence interval with outliers shown as open circles and extreme outliers shown with asterisks.
Table 2 Symptom length in exacerbations shown as median and interquartile range for both treated and untreated episodes

\begin{tabular}{|c|c|c|c|}
\hline Symptom & Treated & Untreated & $P$-value \\
\hline Worse than normal dyspnea & $6(4-13)$ days & $4(2-7)$ days & $<0.001$ \\
\hline \multirow[t]{2}{*}{ Increased sputum volume } & $10(5-18)$ & $6.5(3-12.25)$ & 0.03 \\
\hline & days & days & \\
\hline Increased sputum purulence & $8(4-13)$ days & $6(3-9)$ days & 0.13 \\
\hline
\end{tabular}

Note: The significance of differences between the groups $(P)$ is shown.

\section{Treatment}

Patients received antibiotics or corticosteroids in $81 \%$ of the treated episodes and increased their bronchodilator therapy alone in $19 \%$. When treatment was initiated, it was started with a median of 2 days (IQR 1-4 days) after deterioration of the first symptom. Purulent exacerbations (when sputum color score changed to $\geq 3$ ) lasted significantly longer than nonpurulent episodes $(P<0.001)$, with median values of 14 days (6-26.50) and 7 days (4-11.50), respectively. Antibiotics were used in $90 \%$ purulent exacerbations compared with $67 \%$ of nonpurulent exacerbations; however, time to initiation of treatment for these episodes was not significantly different $(P=0.14)$.

\section{Predictors of treatment}

A forward-entering binary logistic regression model using AT major symptoms and AT type was conducted using the data set of all 233 exacerbations. As shown in Table 3, the numbers of days that the dyspnea symptom was worse than baseline and AT exacerbation type were significant predictors of the initiation of treatment. For every day dyspnea was worse than baseline, the odds of treatment increase by $20 \%$. AT type 1 exacerbations were $11(95 \%$ confidence interval $=3.60-34.67)$ times more likely to be treated than AT type 3 .

The model was statistically significant $\left(\chi^{2}=74.16, d f=5\right.$, $P<0.001)$ and able to classify $71 \%$ of untreated episodes and $74 \%$ of treated episodes correctly with a success rate of $73 \%$.

Tables 4 and 5 show the logistic regression analyses using the same variables as above when the data set was dichotomized to those with and without chronic bronchitis. Both models for predicting treatment in those with and without bronchitis were significant $\left(\chi^{2}=65.60, d f=5, P<0.0001\right.$ and $\chi^{2}=16.19, d f=5, P=0.006$, respectively). In the regression model for patients with chronic bronchitis, the number of days dyspnea and sputum volume was worse than normal were significant predictors of treatment in addition to AT type. For every day dyspnea was worse than normal, the odds of treatment increased by $\sim 25 \%$. For every day sputum volume was worse than normal, the odds of treatment decreased by $\sim 12 \%$. The regression model for patients 
A

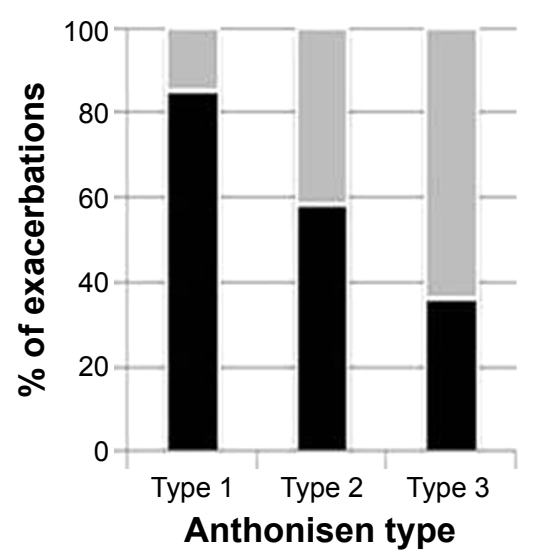

B

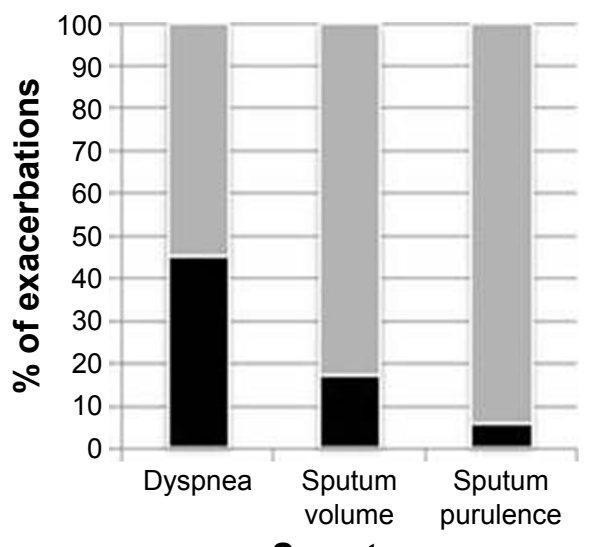

Symptom

Untreated $\mathbf{T}$ Treated

Figure 2 Exacerbation treatment by Anthonisen type and symptom predominance.

Notes: (A) The proportion of exacerbations that were treated is shown for each of the three Anthonisen categories. (B) Anthonisen type 3 exacerbations are shown divided into the proportion of each that were treated episodes; worse than normal dyspnea and increased sputum volume or purulence.

without chronic bronchitis showed the length of abnormal dyspnea as the only significant predictor of treatment, with every day this symptom being worse than normal increasing the odds of treatment by $18 \%$.

\section{Patterns of exacerbations}

Figure 3A shows the analysis of a "typical" treated AT type 1 episode, in which all three major AT symptoms deteriorated at the same time. The left-hand scale indicates the score for each of the AT symptoms. All three symptoms deteriorated on day 1 of the exacerbation and remained worse than normal before dyspnea returned to baseline state on day 4 with sputum volume and color returning to baseline on day 5 .

In addition to classical AT type exacerbations shown in Figure 3A, analysis of the electronic diary cards demonstrated other patterns that occur. Figure 3B demonstrates

Table 3 Variables in the equation for logistic regression of all exacerbations

\begin{tabular}{lllllll}
\hline Variable & B & SE & Sig & $\operatorname{Exp(B)}$ & \multicolumn{2}{l}{$\begin{array}{l}\text { 95\% CI for } \\
\operatorname{Exp(B)}\end{array}$} \\
\cline { 3 - 7 } & & & & & Lower & Upper \\
\hline SOB & 0.19 & 0.04 & 0.00 & 1.20 & 1.11 & 1.30 \\
SpVol & -0.04 & 0.03 & 0.18 & 0.96 & 0.91 & 1.02 \\
SpCol & -0.03 & 0.02 & 0.25 & 0.97 & 0.93 & 1.02 \\
AT type & & & 0.00 & & & \\
AT type I & 2.42 & 0.58 & 0.00 & 11.19 & 3.61 & 34.67 \\
AT type 2 & 1.35 & 0.40 & 0.00 & 3.84 & 1.83 & 8.07 \\
Constant & -1.36 & 0.29 & 0.00 & 0.26 & & \\
\hline
\end{tabular}

Note: The significance $(P)$ of the variable impact is shown as significance. Abbreviations: AT, Anthonisen; $\mathrm{Cl}$, confidence interval; SE, standard error; Sig, significance; $\mathrm{SOB}$, dyspnea score; $\mathrm{SpVol}$, length of those with new or increased sputum volume; $\mathrm{SpCol}$, length of episodes with new or increased sputum purulence. a pattern whereby the patient's baseline sputum volume is "re-set" following the exacerbation and never returned to the pre-exacerbation state. In addition, some patients whose baseline state always included daily sputum production or daily purulent sputum production lost these symptoms following a clear episode of a treated exacerbation (Figure 3C). Figure $\mathrm{S} 1$ demonstrates an exacerbation whereby one symptom oscillates for a number of days before deterioration of all other symptoms.

\section{Discussion}

This study provides unique insight into the characterization of patient symptoms during exacerbations. The analysis of real-time electronic diary cards identified 103 (44\%) untreated (unreported) exacerbations, which is consistent with the observation in a number of other studies indicating

Table 4 Logistic regression of exacerbations in patients with chronic bronchitis

\begin{tabular}{lllllll}
\hline Variable & B & SE & Sig & $\operatorname{Exp(B)}$ & $\begin{array}{l}\text { 95\% Cl for } \\
\text { Exp(B) }\end{array}$ \\
\cline { 3 - 7 } & & & & & Lower & Upper \\
\hline SOB & 0.23 & 0.06 & 0.00 & 1.25 & 1.13 & 1.4 \\
SpVol & -0.12 & 0.05 & 0.01 & 0.88 & 0.81 & 0.96 \\
SpCol & -0.02 & 0.03 & 0.42 & 0.98 & 0.93 & 1.03 \\
AT type & & & 0.00 & & & \\
AT type I & 3.37 & 0.76 & 0.00 & 28.95 & 6.52 & 128.57 \\
AT type 2 & 1.60 & 0.47 & 0.00 & 4.96 & 2.00 & 12.37 \\
Constant & -1.63 & 0.38 & 0.00 & 0.20 & & \\
\hline Abbrevitionyyyyy
\end{tabular}

Abbreviations: AT, Anthonisen; $\mathrm{Cl}$, confidence interval; SE, standard error; Sig, significance; SOB, dyspnea score; $\mathrm{SpVol}$, length of those with new or increased sputum volume; $\mathrm{SpCol}$, length of episodes with new or increased sputum purulence. 
Table 5 Logistic regression of exacerbation episodes in patient without chronic bronchitis

\begin{tabular}{lllllll}
\hline Variable & B & SE & Sig & $\operatorname{Exp(B)}$ & \multicolumn{2}{c}{$\begin{array}{l}\text { 95\% Cl for } \\
\text { Exp(B) }\end{array}$} \\
\cline { 3 - 7 } & & & & & Lower & Upper \\
\hline SOB & 0.16 & 0.08 & 0.03 & 1.18 & 1.01 & 1.36 \\
SpVol & 0.04 & 0.05 & 0.38 & 1.04 & 0.95 & 1.15 \\
SPCol & -0.03 & 0.07 & 0.65 & 0.97 & 0.85 & 1.11 \\
AT type & & & 0.43 & & & \\
AT type I & 1.09 & 1.06 & 0.31 & 2.97 & 0.37 & 23.80 \\
AT type 2 & 0.85 & 0.74 & 0.26 & 2.33 & 0.54 & 10.01 \\
Constant & -1.15 & 0.54 & 0.03 & 0.32 & & \\
\hline
\end{tabular}

Abbreviations: AT, Anthonisen; $\mathrm{Cl}$, confidence interval; $\mathrm{SE}$, standard error; Sig significance; $\mathrm{SOB}$, dyspnea score; $\mathrm{SpV}$ ol, length of those with new or increased sputum volume; $\mathrm{SpCol}$, length of episodes with new or increased sputum purulence.

that unreported exacerbation episodes are common in both "usual" COPD and AATD. ${ }^{6,14}$ This almost certainly indicates a difference in the type and perception of untreated and treated (reported) episodes. It is possible that daily electronic prompting may have an effect on the patterns seen here compared with other studies. However, the patients included in the trial had to have a history of frequent exacerbations as an entry criteria and the frequency of treated and untreated episodes identified is consistent with other studies. This suggests that over-reporting is not an issue and the prompting more likely highlights the real pattern of change of individual symptoms that may be reflected less well in paper diary or recall methodologies.

Here, we have shown that the untreated episodes were significantly shorter than the treated episodes in AATD, consistent with our previous publication. ${ }^{6}$ Untreated episodes were characterized by a significantly shorter duration of dyspnea and were more likely to be AT type 2 or 3 events. Conversely, AT type 1 exacerbations (all major symptoms deteriorate) were more likely to be treated. However, when the AT type 3 exacerbations were characterized by their symptom change, dyspnea AT type 3 events were most likely to receive treatment. This finding supports the fact that the perception of dyspnea by the patients is the most important
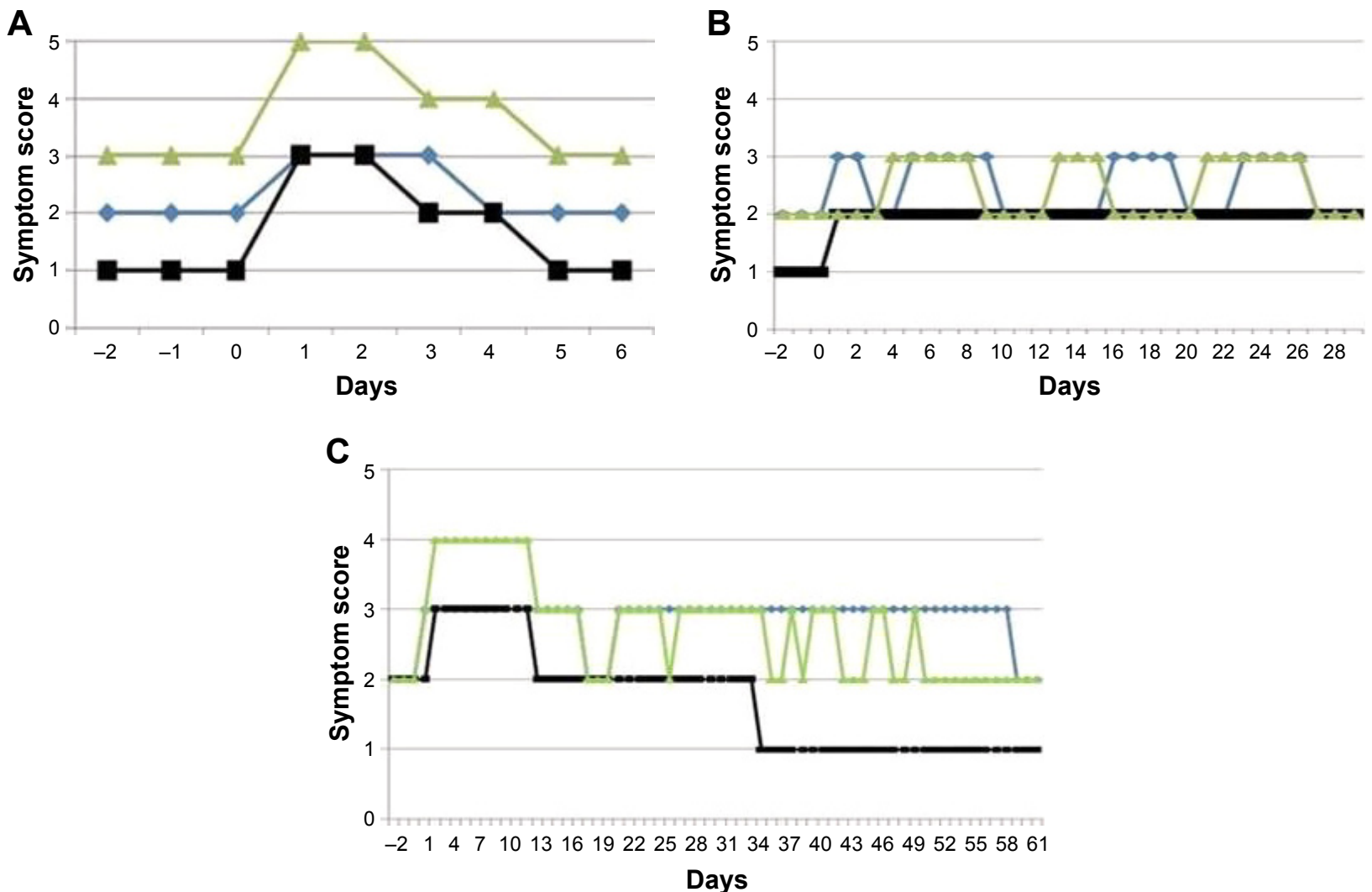

Days

$\rightarrow$ SOB $\rightarrow$ Sputum volume $\rightarrow$ Sputum color

Figure 3 Patterns of exacerbations.

Notes: (A) Classical Anthonisen type I exacerbations SOB, sputum volume, sputum color. (B) Reset baseline with increased sputum production following treated exacerbations. Treatment started on day I and finished on day II. (C) Reset baseline with reduction in sputum volume following a treated exacerbation. Treatment started on day 3 and finished on day 27. Dyspnea was scored on a 4-point scale $(2=$ normal or usual for me, $3=$ worse than usual, $4=$ much worse than usual, and $I=$ better than usual), daily sputum volume was scored as none =(0), I= (up to a teaspoonful), $2=$ (up to a tablespoonful), $3=$ (up to an egg-cupful), $4=$ (more than an egg-cupful), and sputum color was assessed using the Bronkotest color chart ( $\mathrm{I}-2$ being mucoid and 3,4 , and 5 being increasing purulence).

Abbreviation: SOB, dyspnea score. 
indicator for escalation of their treatment. In fact our previous study, which used a scoring system for analyzing exacerbations, weighted dyspnea by a factor of 5 because of its impact on patient symptomatology. ${ }^{6}$

Despite patients being in a clinical trial, the treatment of episodes was not proscribed and predominantly left to the patients' own rescue packs (where present) or local medical practitioners. Interestingly, purulent predominant AT type 3 exacerbations (which are highly proteolytically active) were less likely to be treated in this patient cohort. This is an important observation because in this population, sputum purulence is also classically ${ }^{16}$ associated with bacterial infection. The caveat to this is that the absence of sputum purulence cannot definitively identify a bacterial cause of symptom deterioration and highlights some of the discrepancies in the literature between the relationship of pathogenic bacterial infection and sputum color. ${ }^{17,18}$ Studies have shown that high bacterial loads in respiratory secretions in patients with acute exacerbations of COPD are associated with increased inflammation and the accompanying proteolytic activity. ${ }^{19}$ Whether this contributes directly to declining lung function is unclear; however, these effects are markedly increased in AATD compared with patients with "usual" COPD. ${ }^{20}$ It is probable that untreated purulent episodes are likely to cause proteolytic lung damage and may underlie disease progression, suggesting that treatment should be considered as part of patient education for identifying such episodes. Conversely, many of the type 2 and 3 episodes where sputum purulence was not a feature received antibiotics prescribed by their nontrial practitioners despite its pathophysiological relevance. ${ }^{21}$

The logistic regression analysis further supports dyspnea as the most important AT symptom for the patient in driving treatment. In all analyses, the duration of "worse than normal dyspnea" was a significant predictor of treatment, whereby for every day this symptom remained worse than usual, the odds of treatment increased by $25 \%$. Again AT type 1 exacerbations were more likely to be the predictor of treatment than AT type 3. When the data set was reanalyzed for the presence of chronic bronchitis ( $n=32$ of 43 patients), one of the most interesting observations was that the number of days sputum volume was increased acted as a significant negative predictor of treatment in this cohort. The explanation is unclear, but it is possible that because these patients produce sputum on a daily basis they either become used to it or ignore minor fluctuations as a nuisance rather than an important clinical signal. Clearly further studies are needed to characterize this trait further.
Analysis of patterns of patient symptoms during and after the exacerbation period has shown that these also vary. The general concept is that exacerbations represent defined episodes when symptoms deteriorate from the "normal pattern" for at least 2 consecutive days and then resolve with or without treatment. The real-time data collected here show that symptoms can deteriorate sequentially and resolve sequentially. Furthermore, they can undergo a stuttering pattern of deterioration in one or more symptoms for several days with short periods of stability before a fully recognized exacerbation event occurs. In addition, patients' stable state symptoms, for example, sputum volume or sputum purulence, can be reset in either an upward or downward fashion following recovery from the exacerbation. This has implications in two ways. First, if the symptom baseline becomes persistently lower following therapy, does a return to the previous baseline (ie, a subsequent deterioration) indicate just a late return to normal or should it be considered as another exacerbation that needs treatment thereafter aimed at retaining the symptom baseline as low as possible? Second, does an episode that is higher and does not return to baseline indicate an inadequate type or length of therapy or a progression of disease features? Both scenarios are rarely considered in the management of long-term conditions such as AATD and COPD in general but, in principle, should lead to maintenance of the best possible clinical status.

These observations also raise issues about the use of therapies to treat or prevent exacerbations. Not all exacerbations of COPD are prevented by usual therapies such as long-acting beta agonists, long-acting muscarinic agonist, and even long-term antibiotics. It is inherent in future studies that not only the presence of exacerbations is recorded but also their nature and length. For instance, the observation that treatment with tiotropium or salmeterol/fluticasone changed the characteristics of the exacerbation with the latter requiring more antibiotics in the INSPIRE study has yet to be explored further. ${ }^{22}$ Did the azithromycin study ${ }^{23}$ only prevent purulent episodes or lead to a reset of underlying bacterial load or purulent sputum production? The data reported here suggest that not just the presence of exacerbations needs to be addressed but also the nature and perhaps a more personalized approach to treatment and prevention.

While the AT criteria still remain the fundamental framework by which to classify exacerbations in clinical practice, our research indicates that not only identifying the AT type but also which symptom predominates is important in understanding the exacerbations that patients perceive as needing treatment. This is rarely reported in clinical trials 
and would be important in the design of future studies. As the current study was part of a formal clinical trial, the information focused on identifying the frequency and severity of exacerbations (defined by their treatment) and little extensive data and sample collection was undertaken. The use of the electronic diary has highlighted several features previously unrecorded. The data highlight new patterns that suggest that a more in-depth monitoring is essential to understand the nature, impact, and treatment of these episodes including future mapping against biomarkers to define the episodes in even more detail than is conventionally undertaken, leading toward a more personalized approach to management of individual episodes. This would include, for instance, determining the real effect of sputum purulence in long-term progression and the need to treat such episodes even in the absence of the more immediate impact of dyspnea.

In summary, analysis of real-time electronic diary cards provides valuable information about the characterization of exacerbations. Untreated episodes are common and are significantly shorter in duration than the treated episodes. Dyspnea is the most important single AT symptom in prediction of treatment, and predictors of treatment are different in patients with chronic bronchitis.

\section{Acknowledgment}

Kamada conducted the clinical trial.

\section{Disclosure}

SIE was funded by a noncommercial grant from CSL Behring. The authors report no other conflicts of interest in this work.

\section{References}

1. Vestbo J, Hurd SS, Agusti AG, et al. Global strategy for the diagnosis, management, and prevention of chronic obstructive pulmonary disease: GOLD executive summary. Am J Respir Crit Care Med. 2013;187(4): 347-365.

2. Halbert RJ, Isonaka S, George D, Iqbal A. Interpreting COPD prevalence estimates: what is the true burden of disease? Chest. 2003; 123(5):1684-1692.

3. Seemungal TA, Donaldson GC, Paul EA, Bestall JC, Jeffries DJ, Wedzicha JA. Effect of exacerbation on quality of life in patients with chronic obstructive pulmonary disease. Am J Respir Crit Care Med. 1998; 157(5 Pt 1):1418-1422.

4. Seemungal TA, Hurst JR, Wedzicha JA. Exacerbation rate, health status and mortality in COPD - a review of potential interventions. Int J Chron Obstruct Pulmon Dis. 2009;4:203-223.
5. Connors AF Jr, Dawson NV, Thomas C, et al. Outcomes following acute exacerbation of severe chronic obstructive lung disease. The SUPPORT investigators (Study to Understand Prognoses and Preferences for Outcomes and Risks of Treatments). Am J Respir Crit Care Med. 1996;154(4 Pt 1):959-967.

6. Vijayasaratha K, Stockley RA. Reported and unreported exacerbations of COPD: analysis by diary cards. Chest. 2008;133(1):34-41.

7. Soler-Cataluna JJ, Martinez-Garcia MA, Roman Sanchez P, Salcedo E, Navarro M, Ochando R. Severe acute exacerbations and mortality in patients with chronic obstructive pulmonary disease. Thorax. 2005;60(11):925-931.

8. Donaldson GC, Seemungal TA, Bhowmik A, Wedzicha JA. Relationship between exacerbation frequency and lung function decline in chronic obstructive pulmonary disease. Thorax. 2002;57(10):847-852.

9. Needham M, Stockley RA. Exacerbations in \{alpha\}1-antitrypsin deficiency. Eur Respir J. 2005;25(6):992-1000.

10. Anthonisen NR, Manfreda J, Warren CP, Hershfield ES, Harding GK, Nelson NA. Antibiotic therapy in exacerbations of chronic obstructive pulmonary disease. Ann Intern Med. 1987;106(2):196-204.

11. Aaron SD, Vandemheen KL, Hebert P, et al. Outpatient oral prednisone after emergency treatment of chronic obstructive pulmonary disease. N Engl J Med. 2003;348(26):2618-2625.

12. Cazzola M, MacNee W, Martinez FJ, et al. Outcomes for COPD pharmacological trials: from lung function to biomarkers. Eur Respir J. 2008; 31(2):416-469.

13. Rodriguez-Roisin R. Toward a consensus definition for COPD exacerbations. Chest. 2000;117(5 Supp1 2):398S-401S.

14. Jones PW, Lamarca R, Chuecos F, et al. Characterisation and impact of reported and unreported exacerbations: results from ATTAIN. Eur Respir J. 2014;44(5):1156-1165.

15. Seemungal TA, Donaldson GC, Bhowmik A, Jeffries DJ, Wedzicha JA Time course and recovery of exacerbations in patients with chronic obstructive pulmonary disease. Am J Respir Crit Care Med. 2000; 161(5):1608-1613.

16. Miravitlles M, Espinosa C, Fernandez-Laso E, Martos JA, Maldonado JA, Gallego M. Relationship between bacterial flora in sputum and functional impairment in patients with acute exacerbations of COPD. Study Group of Bacterial Infection in COPD. Chest. 1999; 116(1):40-46.

17. Brusse-Keizer MG, Grotenhuis AJ, Kerstjens HA, et al. Relation of sputum colour to bacterial load in acute exacerbations of COPD. Respir Med. 2009;103(4):601-606.

18. Soler N, Esperatti M, Ewig S, Huerta A, Agusti C, Torres A. Sputum purulence-guided antibiotic use in hospitalised patients with exacerbations of COPD. Eur Respir J. 2012;40(6):1344-1353.

19. Gompertz S, O'Brien C, Bayley DL, Hill SL, Stockley RA. Changes in bronchial inflammation during acute exacerbations of chronic bronchitis. Eur Respir J. 2001;17(6):1112-1119.

20. Gompertz S, Hill AT, Bayley DL, Stockley RA. Effect of expectoration on inflammation in induced sputum in alpha-1-antitrypsin deficiency. Respir Med. 2006;100(6):1094-1099.

21. Stockley RA, O'Brien C, Pye A, Hill SL. Relationship of sputum color to nature and outpatient management of acute exacerbations of COPD. Chest. 2000;117(6):1638-1645.

22. Wedzicha JA, Calverley PM, Seemungal TA, et al. The prevention of chronic obstructive pulmonary disease exacerbations by salmeterol/ fluticasone propionate or tiotropium bromide. Am J Respir Crit Care Med. 2008;177(1):19-26.

23. Albert RK, Connett J, Bailey WC, et al. Azithromycin for prevention of exacerbations of COPD. N Engl J Med. 2011;365(8):689-698. 


\section{Supplementary material}

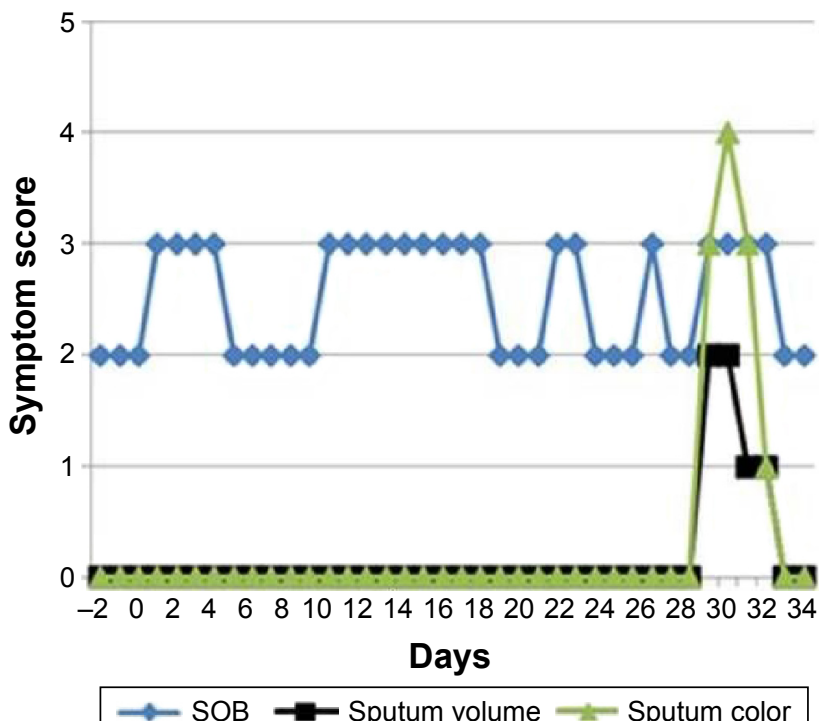

Figure SI Exacerbation in which only one symptom (dyspnea) oscillates for $>3$ weeks before all other major Anthonisen symptoms deteriorate.

Notes: Dyspnea was scored on a 4 -point scale $(2=$ normal or usual for me, $3=$ worse than usual, $4=$ much worse than usual, and I= better than usual), daily sputum volume was scored as none $=(0), I=$ (up to a teaspoonful), 2 = (up to a tablespoonful), $3=$ (up to an egg-cupful), $4=$ (more than an egg-cupful), and sputum color was assessed using the Bronkotest color chart ( $\mathrm{I}-2$ being mucoid and 3, 4, and 5 being increasing purulence).

Abbreviation: SOB, dyspnea score.

\section{Publish your work in this journal}

The International Journal of COPD is an international, peer-reviewed journal of therapeutics and pharmacology focusing on concise rapid reporting of clinical studies and reviews in COPD. Special focus is given to the pathophysiological processes underlying the disease, intervention programs, patient focused education, and self management protocols.
This journal is indexed on PubMed Central, MedLine and CAS. The manuscript management system is completely online and includes a very quick and fair peer-review system, which is all easy to use. Visit $\mathrm{http}: / /$ www.dovepress.com/testimonials.php to read real quotes from published authors. 